\title{
A serious dermatological side effect due to vortioxetine: a case report
}

\author{
Burak Okumus $^{1 \oplus}$, Kader Semra Karatas $^{1 \oplus}$, Asli Enzel Koc ${ }^{1 \oplus}$ \\ ${ }^{1}$ Recep Tayyip Erdogan University, Faculty of Medicine, Department of Psychiatry, Rize - Turkey
}

\begin{abstract}
Major depressive disorder is the second-largest healthcare problem worldwide in terms of illness-induced disability, and second-generation antidepressants are the most commonly used treatments in this disorder. Vortioxetine is a recently introduced antidepressant with a multimodal mechanism of action. Some side effects associated with the use of vortioxetine have been reported. Side effects that do not occur in clinical trials can be reported during phase IV studies. In this case report, we present a patient who developed dermatological adverse reactions following vortioxetine use.
\end{abstract}

Keywords: Ecchymosis, skin eruption, vortioxetine

\section{INTRODUCTION}

Major depressive disorder (MDD) is a fairly common condition that may reduce the quality of life (1). While the biological foundations of MDD have not yet been sufficiently understood, current theories concentrate on questions related to neuroplasticity, neurotrophin signaling, the role of neuroinflammation, hyperactivity of the hypothalamus-hypophysis-adrenal axis, and most importantly," monoaminergic neurotransmission (2). The last hypothesis, emphasizing anomalies in the serotonergic, noradrenergic, and dopaminergic systems, is still a cornerstone in the development of antidepressant drugs. Accordingly, the literature shows that in depressive episodes of intermediate to high severity, antidepressant drugs chosen for treatment are selective serotonin (5-HT) reuptake inhibitors (SSRIs) and serotonin and noradrenaline reuptake inhibitors (SNRIs) $(1,2)$. However, limited efficacy, low remission rates, and undesirable side effects such as weight changes, sexual dysfunctions, and insomnia that can affect compliance with therapy as well as observed resistance to treatment indicate a need for new treatment strategies $(1,3)$. A recent antidepressant with different mechanisms of action for this purpose in clinical use is vortioxetine (1), a multimodal antidepressant with 5-HT carrier inhibitor, 5-HT3, 5-HT7, and 5-HT1D receptor antagonist, 5-HT1B partial agonist, and 5HT1A agonist properties (4). Nonclinical in vivo studies showed that vortioxetine increases the levels of serotonin, noradrenaline, dopamine, acetylcholine, and histamine in certain brain areas (5). Among antidepressants evaluated in a placebo-controlled study, vortioxetine was found to have the greatest effect on psychomotor speed, executive function, and cognitive control (6). It is assumed that vortioxetine used in MDD does not increase fatigue or somnolence reported subjectively by the patients (7). Another advantage in MDD treatment, compared to other SSRIs, is the absence of significant weight gain

How to cite this article: Okumus B, Karatas KS, Enzel Koc A. A serious dermatological side effect due to vortioxetine: a case report. Dusunen Adam The Journal of Psychiatry and Neurological Sciences 2020;33:87-91.

Correspondence: Burak Okumus, MD, Recep Tayyip Erdogan University, Faculty of Medicine, Department of Psychiatry, Rize - Turkey 
and lower sexual dysfunction. With the use of vortioxetine, gastrointestinal side effects (nausea, emesis), dizziness, sweating, headache, dryness of the mouth, hyponatremia, and sleep disturbances have been reported (5). Drug development studies with substances produced for clinical use undertaken prior to phase IV will not be able to detect all adverse drug reactions (ADR), given that a sufficient population will not be reached. Hence, the determination of ADRs and any problems related to drug safety are very important (8). Early identification of ADRs is crucial for drug management, as these reactions constitute one of the main reasons for morbidity, recourse to emergency services, and hospitalization. The dose of vortioxetine recommended in MDD therapy is $5-20 \mathrm{mg} /$ day (5). Possible dermatological side effects of vortioxetine are redness of the face, neck, arms, and upper chest region, red or purple spots, and itching of the skin (9). Our case presentation aims to report that a patient using vortioxetine $20 \mathrm{mg} / \mathrm{day}$ may develop serious dermatological side effects, beginning with petechial eruptions followed by itching, edema, and ecchymosis.

\section{CASE}

A 34-year-old female married patient presented to our psychiatry clinic with avolition, loss of energy, feelings of guilt, insomnia, loss of appetite, reduced attention and

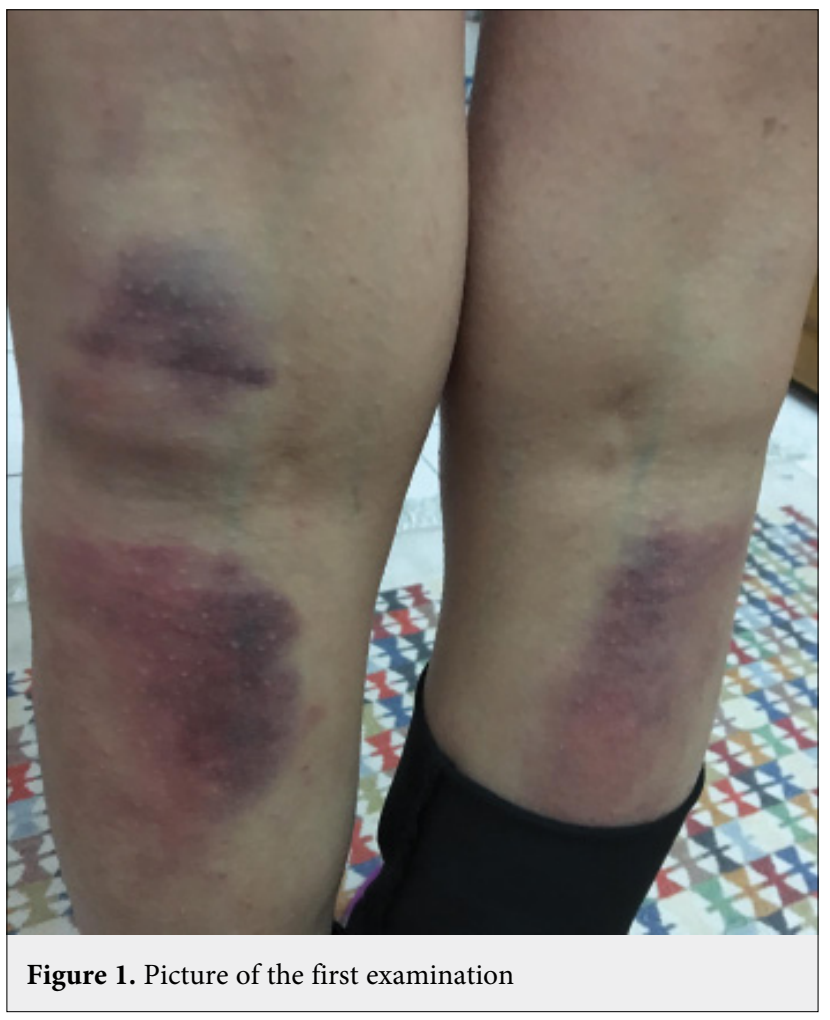

concentration, and occasional outbursts of anger. In the detailed anamnesis, she stated that she had first presented to a psychiatric service 17 years previously with outbursts of anger, avolition, and loss of appetite with related unintentional weight loss. According to her report, she had been treated with sertraline $50 \mathrm{mg} /$ day and mirtazapine $15 \mathrm{mg} /$ day and followed this therapy regularly for 2 years. Ten years ago, she had again suffered similar complaints and received treatment with escitalopram $10 \mathrm{mg}$ /day and mirtazapine $15 \mathrm{mg} /$ day for 9 months. When she suffered a relapse 6 years ago, she used venlafaxine $300 \mathrm{mg} /$ day for 2 years. She stated that these treatments had been partly useful. After 4 years without treatment, the patient presented to our clinic 2 months ago with complaints of low mood, concentration problems, outbursts of anger, reduced amount of sleep, and loss of appetite. After clinical interview, the patient was diagnosed with MDD according to Diagnostic and Statistical Manual of Mental Disorders Fifth Edition (DSM-5). Treatment was started with vortioxetine $5 \mathrm{mg} /$ day for the first 4 days and subsequently vortioxetine $10 \mathrm{mg} /$ day. After 2 months of treatment with vortioxetine $10 \mathrm{mg} /$ day, the patient reported a partial improvement of her complaints, and the dose of vortioxetine was increased to $20 \mathrm{mg} /$ day. Four days later, with onset of petechial eruptions followed by itching and ecchymoses bilaterally in the popliteal regions, the patient returned to our service (Figure 1). Her blood values were not pathological. Further tests and examinations undertaken in the clinics of dermatology, hematology, and cardiovascular surgery did not detect any other underlying causes. Supposing that the lesions were drugrelated, the administration was discontinued. The patient's dermatological lesions declined within 4 days (Figure 2) and resolved entirely after 2 weeks (Figure 3).

Personal history: The patient had been delivered in hospital by natural birth without complications. No diseases during infancy were recorded. Toilet training, walking, and talking had developed in normal time. Menarche had occurred at the age of 10 years. She graduated from university. According to communications received from her and her family, social relations were good except during disease episodes. She had been married twice, with one child from the first marriage, while the second marriage had been contracted 3 months ago. It was learned that she had not used alcohol or substances, did not suffer from any other systemic diseases, and had been diagnosed for similar complaints with MDD.

Family history: It was learned that the patient's mother, her maternal grandmother, and 1 sister had a 


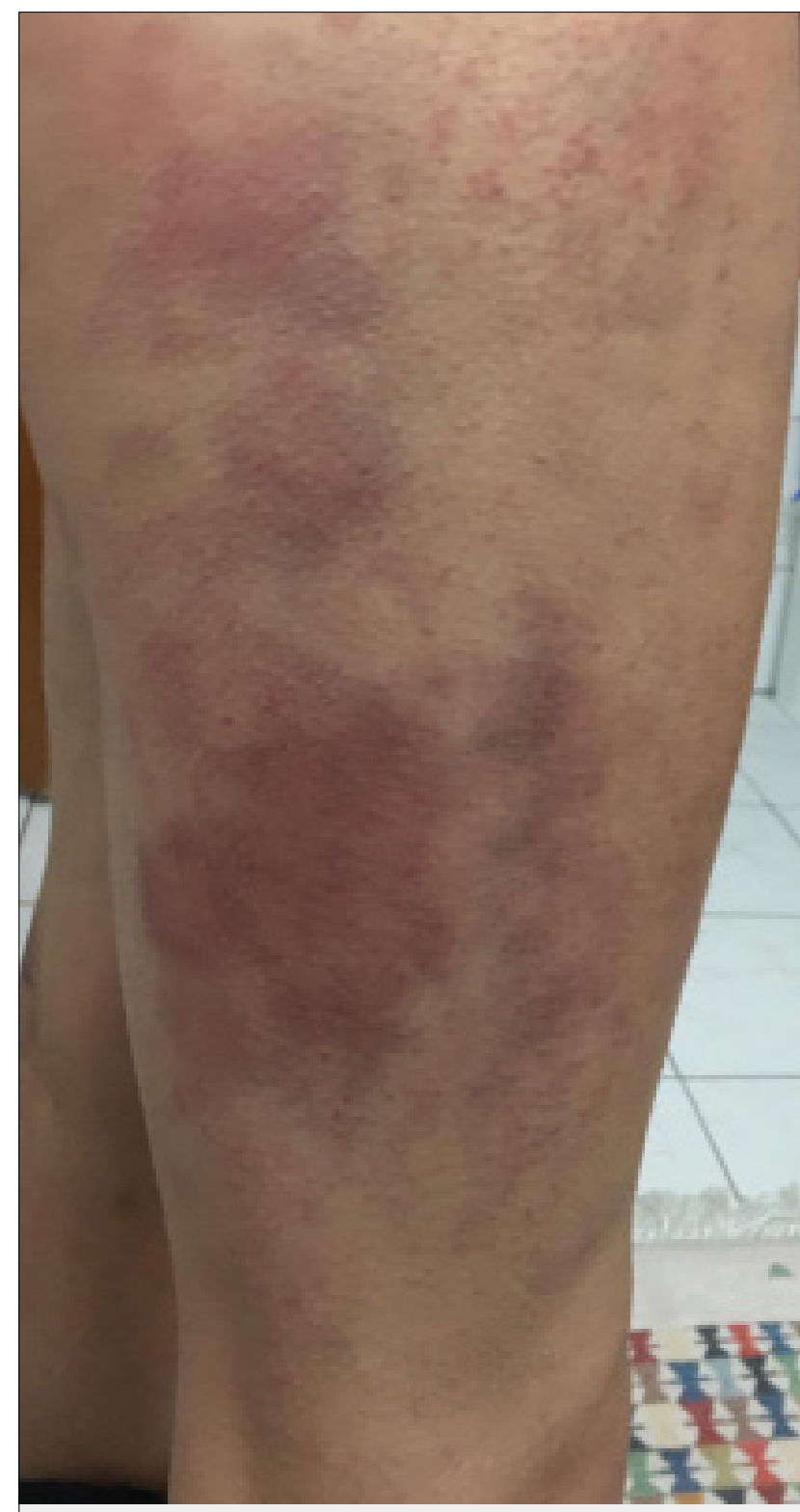

Figure 2. Picture of the second examination four days after discontinuation of the medicine

history of repeated episodes of AD drug use. Her father had died of a hematological bone marrow malignancy with unknown specification.

Physical examination and laboratory results: Vital signs, neurological and other systemic examinations were within normal limits. No pathological results were found in further examinations carried out in consultations with hematology, dermatology, and cardiovascular surgery. Height: $160 \mathrm{~cm}$, weight: $48 \mathrm{~kg}$, body mass index (BMI): $18.75 \mathrm{~kg} / \mathrm{m}^{2}$.

Laboratory results: Hematological and biochemical parameters were in the normal range, hormone test results were normal (thyroid function tests [TSH, T3, T4], parathormone, ceruloplasmin, vasculitis markers,

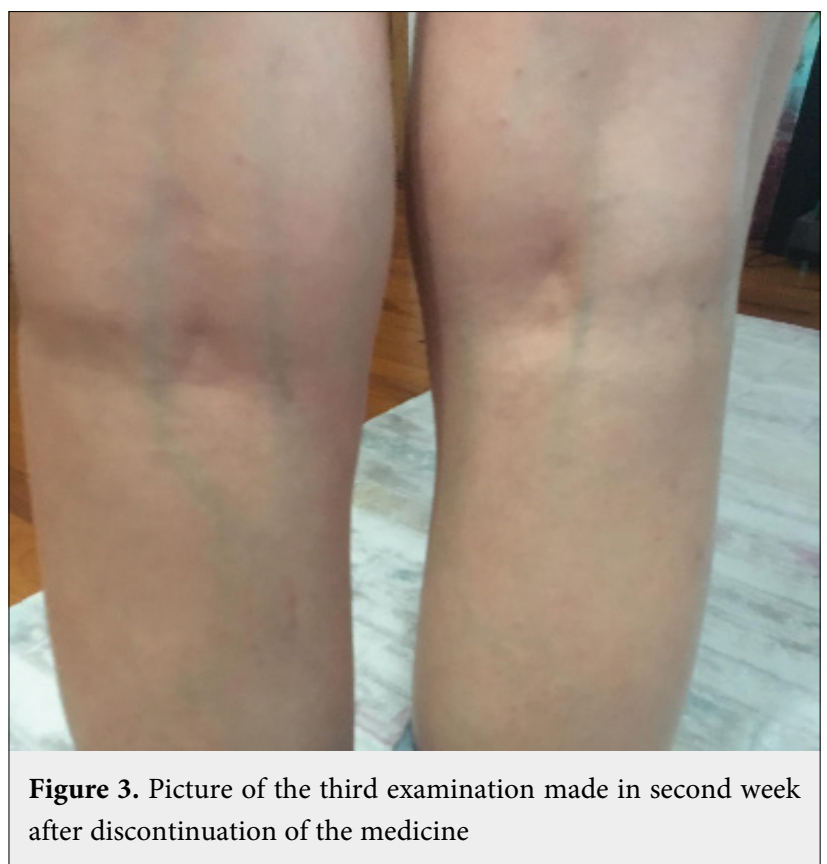

vitamin B12, ferritin, and folic acid in normal limits). Prothrombin time, active partial thromboplastin time, fibrinogen level, platelet function tests, bleeding time, and peripheral smear test results were normal.

No pathologies were found in cranial magnetic resonance imaging. The electroencephalogram showed waking rhythms in normal limits.

Mental state examination: In the outer appearance, the patient's self-care seemed partly reduced and she seemed thin. The patient appeared normal for her age, was willing to participate in the interview, though the amount of speech was reduced. Her mood was depressed, her affect dysphoric. She was conscious, oriented, and cooperative. No pathologies were detected in her perception. In her thought content, ruminative concentration consistent with the clinical picture and side effects was found. Recall problems in short-term memory and reduced attention were determined. Abstract reasoning, reality assessment and judgment were normal. In expressive behaviors, psychomotor retardation and need for help were observed.

\section{DISCUSSION}

Determining rare adverse effects of drugs introduced into clinical use during phase IV trials is required in order to monitor known risks more safely and draw up therapeutic guidelines for pediatric and other clinical populations. At this stage, the candidate substance's real-world effectiveness and side effects undetected during clinical trials can be determined. As clinical 
trials are carried out under very specific conditions, the incidence of side effects may not reflect the rates seen in clinical application. There are studies indicating that among the psychotropic drugs introduced with great hopes into clinical use during the past 20 years, the effectiveness of vortioxetine is better than that of other SSRIs (5). In particular, the avoidance of significant weight gain, lower sexual dysfunction, and the absence of fatigue and somnolence reported subjectively by the patients suggest that vortioxetine may be advantageous in the treatment of $\operatorname{MDD}(5,7)$. However, in the product monograph of the drug only itching has been reported (5). A literature review of past studies found that antidepressant-related skin reactions usually manifest as slight drug-related exanthemas, while life-threatening systemic reactions may also occur rarely. Among the severe dermatological complications related to the use of antidepressants, we may list erythema multiforme (EM), Stevens-Johnson syndrome (SJS), toxic epidermal necrolysis (TEN), acute generalized exanthematous pustulosis (AGEP), and drug-induced hypersensitivity syndrome (DIHS) (10). In the last few years, psychiatrists and dermatologists collaborating in the treatment of similar situations or other disorders have created psychodermatology as an important field of study in the consultation liaison within psychiatry (11). From this perspective, in the literature the antidepressants mianserin, sertraline, trazodone, and bupropion have been identified related to $\operatorname{EM}(12,13)$. First indications for the occurrence of SJS or TEN after the use of antidepressant drugs were obtained in the 1990s, drawing attention to substances like fluoxetine and sertraline from the group of SSRIs. In 1994, the first patient developing severe TEN after starting paroxetine was reported. In the last few years, new reports regarding the possibility of developing SJS symptoms after the use of mirtazapine were published (10). The dermatological consultation in our case, requested in view of possible SJS, did not result in relevant clinical findings; however, it suggested the possibility of a druginduced dermatological side effect. In the literature, sertraline and amoxapine are listed among the antidepressants suspected of being related with AGEP. Past studies specified the potential for DIHS alongside systemic symptoms after the use of citalopram, fluoxetine, and amitriptyline (10). While reports about ecchymosis as a result of sertraline, venlafaxine, and escitalopram are found in the literature, case reports regarding vortioxetine are limited (14-17). Particularly after the use of serotonin reuptake inhibitors as antidepressants, abnormal bleeding has been reported
(18). Hematological side effects of these drugs are not rare, with vaginal bleedings, epistaxis, purpura, hematuria, intracranial bleedings, and rectal bleeding being the most frequently seen findings. The molecular mechanisms underlying these hematological side effects include the peripheral effects of serotonin, especially a disturbance of platelet aggregation and of the normal process of vascular smooth muscle tonus (19). In addition, noradrenaline reuptake inhibitors (duloxetine, milnacipran and venlafaxine) may increase the risk of bleeding due to drug-induced increase of blood pressure. Noradrenaline and serotonin reuptake inhibitors (duloxetine and venlafaxine) may involve a higher risk of bleeding than noradrenaline reuptake inhibition on its own (20). The antidepressant effect of vortioxetine, on the other hand, is achieved through modulation of neurotransmission in a number of systems, including noradrenalin, dopamine, acetylcholine, histamine, glutamate, and $\gamma$-aminobutyric acid systems (20).

In order to establish a hematological side effect caused by a psychotropic drug, the possibility of an underlying functional platelet disorder needs to be excluded. In our case, hematological parameters and peripheral smears were with within normal limits (20). As the lesions resolved after discontinuing the drug without the need for additional treatment, we can assume that the present picture was related to vortioxetine and the dose increase from 10 to $20 \mathrm{mg} /$ day.

The reported incidence of severe adverse events with vortioxetine is $2.9 \%$ for doses between 5 and $10 \mathrm{mg}$ /day and $2.2 \%$ for vortioxetine doses of $15-20 \mathrm{mg} /$ day (21). When reviewing the literature, we found 2 case reports where dermatological findings resolved with the discontinuation of vortioxetine, one after the development of edema, petechiae, and ecchymosis in the $3 \mathrm{rd}$ month of vortioxetine treatment with $10 \mathrm{mg}$ and one with excessive itching in the 5th week of $5 \mathrm{mg}$ vortioxetine (5). Our case developing petechial eruptions and subsequent itching with secondary ecchymotic lesions on day 4 after increasing the daily dose of vortioxetine from 10 to $20 \mathrm{mg}$ is not in line with findings in the literature suggesting that serious side effects are seen less in treatment with higher doses of vortioxetine. The observation of similar side effects with different dosages emphasizes the importance of interpersonal differences in the treatment of MDD.

To conclude, vortioxetine is an alternative drug in the therapy of treatment-resistant MDD. However, with the increasing use of vortioxetine in MDD treatment of for other clinical reasons, the identification and management 
of serious side effects requires clinicians' attention. It is important for clinicians to be attentive regarding drugs like vortioxetine currently introduced into clinical use and to contribute to the relevant literature.

\begin{tabular}{|c|c|c|}
\hline \multicolumn{2}{|c|}{ Contribution Categories } & \multirow{2}{*}{\begin{tabular}{|l} 
Author Initials \\
B.O., A.E.K., K.S.K.
\end{tabular}} \\
\hline \multirow{4}{*}{ Category 1} & Concept/Design & \\
\hline & Literature review & B.O., A.E.K., K.S.K. \\
\hline & Data analysis/Interpretation & B.O. \\
\hline & Case follow-up (if applicable) & B.O. \\
\hline \multirow{2}{*}{ Category 2} & Drafting manuscript & B.O. \\
\hline & Critical revision of manuscript & B.O., K.S.K., A.E.K. \\
\hline Category 3 & Final approval and accountability & B.O., A.E.K., K.S.K. \\
\hline \multirow{2}{*}{ Other } & Technical or material support & B.O. \\
\hline & Supervision & K.S.K. \\
\hline
\end{tabular}

Informed Consent: Written informed consent was obtained from the patients.

Peer-review: Externally peer-reviewed.

Conflict of Interest: The authors declared no conflict of interest.

Financial Disclosure: None declared.

\section{REFERENCES}

1. Sagud M, Nikolac Perkovic M, Vuksan-Cusa B, Maravic A, Svob Strac D, Mihaljevic Peles A, et al. A prospective, longitudinal study of platelet serotonin and plasma brain-derived neurotrophic factor concentrations in major depression: effects of vortioxetine treatment. Psychopharmacology (Berl) 2016; 233:3259-3267.

2. Sowa-Kućma M, Pańczyszyn-Trzewik P, Misztak P, Jaeschke RR, Sendek K, Styczeń K, et al. Vortioxetine: a review of the pharmacology and clinical profile of the novel antidepressant. Pharmacol Rep 2017; 69:595-601. [CrossRef]

3. Jacobsen PL, Mahableshwarkar AR, Serenko M, Chan S, Trivedi $\mathrm{MH}$. A randomized, double-blind, placebo-controlled study of the efficacy and safety of vortioxetine $10 \mathrm{mg}$ and $20 \mathrm{mg}$ in adults with major depressive disorder. J Clin Psychiatry 2015; 76:575582. [CrossRef]

4. Baldwin DS, Chrones L, Florea I, Nielsen R, Nomikos GG, Palo $\mathrm{W}$, et al. The safety and tolerability of vortioxetine: Analysis of data from randomized placebo-controlled trials and open-label extension studies. J Psychopharmacol 2016; 30:242-252. [CrossRef]

5. Cetin M, Kose S. Serious dermatological adverse effects of vortioxetine: Two cases. Psychiatry and Clinical Psychopharmacology 2018; 28:355-357. [CrossRef]

6. Rosenblat JD, Kakar R, McIntyre RS. The cognitive effects of antidepressants in major depressive disorder: a systematic review and meta-analysis of randomized clinical trials. Int J Neuropsychopharmacol 2015; 19. [CrossRef]
7. Theunissen EL, Street D, Højer AM, Vermeeren A, van Oers A, Ramaekers JG. A randomized trial on the acute and steady-state effects of a new antidepressant, vortioxetine (Lu AA21004), on actual driving and cognition. Clin Pharmacol Ther 2013; 93:493501. [CrossRef]

8. Subeesh V, Singh H, Maheswari E, Beulah E. Novel adverse events of vortioxetine: a disproportionality analysis in USFDA adverse event reporting system database. Asian J Psychiatr 2017; 30:152-156. [CrossRef]

9. Ay R, Aytas O. Acneiform eruption associated with the use of vortioxetine. Psychiatry and Clinical Psychopharmacology 2019; 29:226-228. (Turkish) [CrossRef]

10. Herstowska M, Komorowska O, Cubała WJ, JakuszkowiakWojten K, Gałuszko-Węgielnik M, Landowski J. Severe skin complications in patients treated with antidepressants: a literature review. Postepy Dermatol Alergol 2014; 31:92-97. [CrossRef]

11. Shah B, Levenson JL. Use of psychotropic drugs in the dermatology patient: when to start and stop? Clin Dermatol 2018; 36:748-755. [CrossRef]

12. Ford HE, Jenike MA. Erythema multiforme associated with trazodone therapy. J Clin Psychiatry 1985; 46:294-295.

13. Khan MAU, Fitzgerald K. Low dose sertraline induced erythema multiforme in a child. J Child Adolesc Psychopharmacol 2012; 22:179-180. [CrossRef]

14. Kohn S, Labbate LA. Venlafaxine and Ecchymosis. Can J Psychiatry 1997; 42:91. [CrossRef]

15. Sarma A, Horne MK 3rd. Venlafaxine induced ecchymoses and impaired platelet aggregation. Eur J Haematol 2006; 77:533-537.

16. Kocbiyik S, Batmaz S, Turhan L, Yuncu OA, Caykoylu A. Resolution of ecchymotic lesions due to fluoxetine and escitalopram after introduction of reboxetine. Cukurova Med J 2015; 40(Suppl 1):90-93. (Turkish) [CrossRef]

17. Calhoun JW, Calhoun DD. Prolonged bleeding time in a patient treated with sertraline. Am J Psychiatry 1996; 153:443. [CrossRef]

18. Vandel P, Vandel S, Kantelip JP. SSRI induced bleeding: two case reports. Therapie 2001; 56:445-447.

19. Akbulut S, Yagmur Y, Gumus S, Babur M. Breast ecchymosis: Unusual complication of an antidepressant agent. Int J Surg Case Rep 2014; 5:129-130. [CrossRef]

20. Gahr M, Zeiss R, Lang D, Connemann BJ, Hiemke C, Muche $\mathrm{R}$, et al. Association between haemorrhages and treatment with selective and non-selective serotonergic antidepressants: Possible implications of quantitative signal detection. Psychiatry Res 2015; 229:257-263. [CrossRef]

21. Salagre E, Grande I, Solé B, Sanchez-Moreno J, Vieta E. Vortioxetine: A new alternative for the treatment of major depressive disorder. Rev Psiquiatr Salud Ment 2018; 11:48-59. 